\section{Qualidade de vida em pessoas com lesão medular traumática: um estudo com o WHOQOL-bref}

\section{Quality of live in people with traumatic spinal cord injury: a study with WHOQOL-bref}

\section{Resumo}

Objetivos: Conhecer a percepção de qualidade de vida em pessoas com lesão medular traumática, utilizando a metodologia adotada pela Organização Mundial de Saúde (OMS). Métodos: Trata-se de uma pesquisa transversal, na qual foi realizado um estudo descritivo para conhecer as características sociodemográficas e a distribuição das lesões medulares traumáticas na amostra estudada. Em seguida, com o intuito de conhecer a percepção da qualidade de vida dessa população, utilizou-se o WHOQOL - bref. As análises estatísticas realizadas incluíram análises descritivas de freqüência, tendência central e dispersão e análise inferencial de comparação entre os domínios. O estudo transcorreu de janeiro de 2005 a maio de 2006 e foi desenvolvido com pessoas no momento de sua admissão no programa de reabilitação do Hospital Sarah Brasília. Utilizou-se uma amostra de conveniência, com 111 entrevistados. Resultados: Os indivíduos estudados são, em sua maioria, homens jovens, solteiros, possuidores do ensino fundamental e vítimas de acidentes de trânsito e de armas de fogo. A percepção da qualidade de vida demonstrou que os domínios que refletiram os piores escores de avaliação estavam relacionados ao meio ambiente e à saúde física; e os mais bem avaliados estavam ligados à saúde psicológica e às relações sociais. Conclusão: $\mathrm{O}$ estudo permitiu conhecer a percepção de qualidade de vida do grupo pesquisado. Recomenda-se estudo que inclua tetraplégicos e lesados medulares não traumáticos, com o intuito de se obter uma avaliação mais global.

Palavras-chave: Qualidade de vida. Traumatismos da medula espinhal. Paraplegia. Ajustamento social.

Aprovado pelo Comitê de Ética em Pesquisa da Associação das Pioneiras Sociais - Rede SARAH de Hospitais de Reabilitação - 26/09/06 
Abstract

Objectives: To find the perception of quality of life in people with traumatic spinal cord injury using the methodology adopted by the World Health Organization (WHO). Methods: This was a cross-sectional study in which a descriptive study was first carried out to better understand the socio-demographic characteristics and the distribution of traumatic spinal cord injury in the sample studied. Afterwards, the WHOQOL-bref was used in order to find the perception of quality of life of that population. Statistical analyses carried out included descriptive analysis of frequency, central trend and dispersion, and inferential analysis of comparison between domains. The study was carried out from January 2005 to May 2006, and included individuals upon their admission to the rehabilitation program of Sarah Hospital in Brasilia. A convenience sample of 111 interviewees was utilized. Results: The individuals who participated in the study were predominantly single young men with elementary-level schooling and who were victims of traffic or firearms accidents. The quality of life study showed that the domains that had the worst evaluation scores were related to environment and physical health, and the best scores were related to psychological health and social relationships. Conclusion: The study provided an overview of the perception of quality of life of the studied group. A study that includes quadriplegics and non-traumatic spinal cord injured individuals is recommended in order to obtain a more general evaluation.

Keywords: Quality of life; spinal cord injury; paraplegics; social adjustment.

\section{Introdução}

As lesões medulares são cada vez mais freqüentes devido, principalmente, ao aumento da violência urbana. Os acidentes de trânsito e os ferimentos por arma de fogo são suas causas mais comuns. O traumatismo da medula pode resultar em alterações das funções motora, sensitiva e autônoma, implicando perda parcial ou total dos movimentos voluntários ou da sensibilidade (tátil, dolorosa e profunda) em membros superiores e/ou inferiores e alterações no funcionamento dos sistemas urinário, intestinal, respiratório, circulatório, sexual e reprodutivo ${ }^{1}$.

As lesões medulares, devido à sua gravidade e irreversibilidade, exigem, para melhoria da qualidade de vida dos indivíduos que sofreram esse trauma, um programa de reabilitação longo e que, na maioria das vezes, não leva à cura, mas auxilia na adaptação a uma nova vida. As seqüelas e as dificuldades que essas pessoas enfrentam para retornar à sua vida familiar e social interferem na sua qualidade de vida e são um desafio aos profissionais de um programa de reabilitação ${ }^{1}$.

Nas últimas décadas, o constructo qualidade de vida passou a ser estudado em diversas áreas do conhecimento humano. Conceitos, metodologias de pesquisa e motivos para medir qualidade de vida passaram a ser temas de interesse de médicos, psicólogos, cientistas sociais e filósofos, entre outros ${ }^{2}$. No contexto médico, a mensuração da qualidade de vida surgiu como uma forma de valorizar as percepções do paciente a respeito de vários aspectos da sua vida, e não meramente avaliar seu estado de saúde.

Trata-se de uma abordagem centrada na percepção do indivíduo sobre seu funcionamento em diversas áreas da vida, como, por exemplo, aspectos físicos, ocupacionais, psicológicos e sociais. A qualidade de vida pode mudar ao longo do tempo, de forma global ou em algumas áreas da vida do sujeito ${ }^{3}$. Mas essa expressão, tão debatida entre pesquisadores de 
diversas áreas, e que ocupa cada vez mais espaço na sociedade e na política de saúde, não tem uma definição universal. Nas diversas abordagens sobre o tema, são adotados diferentes conceitos, modelos teóricos e instrumentos de mensuração².

Para a Organização Mundial de Saúde (OMS), qualidade de vida é "a percepção do indivíduo de sua posição na vida, no contexto da cultura e sistema de valores nos quais ele vive e em relação aos seus objetivos, expectativas, padrões e preocupações" ${ }^{4}$. Esse conceito foi construído na década de 1990, a partir de um projeto multicêntrico. O projeto também originou o instrumento World Health Organization Quality of Life - 100 (WHOQOL-100) e sua versão abreviada o WHOQOL-bref ${ }^{5}$. O objetivo do projeto foi construir um conceito e instrumentos que possuíssem uma abordagem transcultural e que contemplassem três aspectos referentes ao constructo qualidade de vida: subjetividade (percepção do indivíduo sobre sua vida); multidimensionalidade (o construto abrange várias dimensões da vida); e presença de elementos de avaliação tanto positivos quanto negativos ${ }^{6,7}$.

No Brasil, a qualidade de vida tem sido pesquisada, especialmente, em condições crônicas de saúde-doença ${ }^{8-12}$ e como adjuvante na análise de intervenções terapêuticas $^{13}$. Estudos com pessoas que sofreram lesão da medula espinhal praticamente inexistem. Até o momento, foi publicado apenas um trabalho ${ }^{14}$. Contudo, as seqüelas decorrentes desse trauma, assim como as dificuldades sociais, são realidades presentes na vida dessas pessoas e podem interferir em sua qualidade de vida.

$O$ presente estudo tem como objetivo conhecer a percepção de qualidade de vida de pessoas com lesão medular, a partir da visão de indivíduos com paraplegia traumática, entrevistados no momento de sua admissão para participar do programa de reabilitação do Hospital Sarah Brasília - centro. Esse conhecimento poderá subsidiar intervenções durante o processo de reabilitação para manter ou melhorar a qualidade de vida dessa população. Conhecer as dimensões avaliadas de forma positiva e negativa permitirá verificar em que áreas são necessárias mudanças, visando a melhoria da qualidade de vida dos entrevistados e a sua mais adequada inserção familiar e social.

$\mathrm{O}$ instrumento utilizado na pesquisa foi o WHOQOL-bref. A escolha desta ferramenta para avaliar a qualidade de vida de pessoas com paraplegia traumática ocorreu devido ao fato de ser um instrumento de avaliação que não se baseia na doença ou lesão. Já foi utilizado e validado no Brasil para algumas patologias ${ }^{7}$ e em idosos ${ }^{15}$, mas ainda não havia sido utilizado em indivíduos com lesão medular. Além disso, inexiste um instrumento específico, testado e validado para estudar qualidade de vida em pessoas que sofreram esse trauma, na população brasileira.

\section{Método}

Trata-se de uma pesquisa observacional de corte transversal. Num primeiro momento foi realizado um estudo descritivo, com o objetivo de conhecer as características sociodemográficas e a distribuição das lesões medulares traumáticas na população estudada. Na seqüência, foi estudada a qualidade de vida utilizando-se o instrumento geral de medida de qualidade de vida da OMS (WHOQOL - bref).

O presente estudo foi realizado no período de janeiro de 2005 a maio de 2006, e foi desenvolvido no Hospital Sarah Brasília - centro. Os participantes da pesquisa foram entrevistados no momento de sua admissão para participar do programa de reabilitação.

A população alvo do estudo foi composta por indivíduos com paraplegia traumática. Foi utilizada uma amostra de conveniência de casos consecutivos. O cálculo amostral foi realizado a partir dos dados referentes ao número de indivíduos com esse trauma, admitidos para participar do programa de reabilitação nos últimos cinco anos $^{16}$. Esses números variaram em torno de 150 pessoas. Com base nesse dado, 
uma amostra com 95\% de confiança, erro máximo igual a $5 \%$ e considerando uma estimativa da proporção igual a 50\% (variância máxima), foi obtido um tamanho de amostra igual a 109 indivíduos. Assim, a amostra ora estudada contou com 111 entrevistados, de forma a atender ao requisito estatístico de validade do estudo.

As características dos indivíduos incluídos na amostra foram: pessoas com lesão medular traumática, com paraplegia, de ambos os sexos, com idade igual ou superior a 16 anos, admitidas para participar do programa de reabilitação do Hospital Sarah Brasília - centro, pela primeira vez. Os critérios de exclusão foram possuir lesão medular de causa não traumática, já ter participado do programa de reabilitação ou possuir comorbidades. A amostra foi composta por todos os indivíduos que atendiam aos critérios de inclusão, que foram admitidos no período estudado e que aceitaram participar da pesquisa.

O WHOQOL-bref, utilizado para avaliar qualidade de vida de populações adultas, contém 26 perguntas, das quais 24 são distribuídas em quatro domínios: físico, psicológico, relações sociais e meio-ambiente. Os domínios são representados por várias facetas e suas questões foram formuladas para uma escala de respostas do tipo Likert, com escala de intensidade (nada-extremamente), capacidade (nadacompletamente), freqüência (nunca-sempre) e avaliação (muito insatisfeito-muito satisfeito; muito ruim-muito bom). Além dos quatro domínios, o instrumento apresenta duas questões gerais: uma faz referência à percepção da qualidade de vida e a outra à satisfação com a saúde ${ }^{7}$.

Os dados foram analisados utilizandose o programa estatístico SPSS 13. As análises estatísticas realizadas incluíram análises descritivas de freqüência, tendência central e dispersão e análise inferencial de comparação entre os domínios. A partir dos valores obtidos para cada uma das vinte e quatro facetas que compõem os quatro domínios foram obtidas as médias das respostas, o que possibilitou verificar quais facetas foram avaliadas positivamente e quais foram negativamente.

O instrumento da OMS não prevê conceitualmente que se possa utilizar o escore global de qualidade de vida. Então, são calculados os escores de avaliação de cada um dos quatro domínios. O valor mínimo dos escores de cada domínio é zero e o valor máximo 100 . O escore de cada domínio é obtido em uma escala positiva, isto é, quanto mais alto o escore, melhor a qualidade de vida naquele domínio.

Com a finalidade de comparar os domínios foram realizadas Análise de Variância (Anova) e Teste de Turkey. A primeira com objetivo de verificar se havia diferença entre os domínios e o segundo para conhecer quais domínios apresentavam diferenças estatisticamente significativas.

Cumprindo a Resolução no. 196/96, do Ministério da Saúde, que versa sobre Pesquisa Envolvendo Seres Humanos no Bra$\mathrm{sil}^{17}$, o projeto do presente estudo foi submetido ao Comitê de Ética na Pesquisa da Rede Sarah de Hospitais de Reabilitação (Processo CEP/APS no. 010/04) e o estudo só teve início após sua aprovação. Todos os participantes foram esclarecidos sobre os objetivos e metodologias adotadas e assinaram o Termo de Consentimento Livre e Esclarecido (TCLE). No caso de menores de idade, o TCLE também foi assinado pelo responsável.

\section{Resultados}

O perfil epidemiológico e clínico da amostra estudada está descrito na Tabela 1.

É importante ressaltar o fato de haver uma supremacia masculina nas vítimas de lesões medulares traumáticas. A preponderância dos homens se fez notar ao se analisar a razão de sexos que, na amostra estudada, foi de 5,5 homens para cada mulher.

Outro dado a ser observado é a média de idade dos indivíduos analisados, que ficou em 31,3 anos, com mediana de 28 anos e desvio padrão de 11 anos. A idade mínima dos entrevistados foi de 16 anos e a máxima 59 anos. 
Em termos de procedência, observouse que o Hospital Sarah Brasília - centro recebe pacientes de quase todos os Estados da Federação. Os Estados mais repre-

Tabela 1 - Perfil epidemiológico e clínico das pessoas com lesão medular traumática

Table 1 - Epidemiological and clinical profile of individuals with traumatic cord injury

\begin{tabular}{|c|c|c|}
\hline Características & iência & $\%$ \\
\hline \multicolumn{3}{|l|}{ Gênero } \\
\hline Homens & 94 & 84,7 \\
\hline Mulheres & 17 & 15,3 \\
\hline \multicolumn{3}{|l|}{ Faixa Etária (anos) } \\
\hline até 20 & 23 & 20,7 \\
\hline $21-30$ & 38 & 34,2 \\
\hline $31-40$ & 25 & 22,6 \\
\hline $41-50$ & 17 & 15,3 \\
\hline Acima de 50 & 8 & 7,2 \\
\hline \multicolumn{3}{|l|}{ Situação Conjugal } \\
\hline Vive sem parceiro / a & 68 & 61,3 \\
\hline Vive com parceiro / a & 43 & 38,7 \\
\hline \multicolumn{3}{|l|}{ Escolaridade } \\
\hline Ensino fundamental & 59 & 53,2 \\
\hline Ensino médio & 33 & 29,7 \\
\hline Ensino superior & 19 & 17,1 \\
\hline \multicolumn{3}{|l|}{ Ocupação } \\
\hline Trabalha & 83 & 74,8 \\
\hline Desempregado & 3 & 2,7 \\
\hline Outros $^{1}$ & 25 & 22,5 \\
\hline \multicolumn{3}{|l|}{ Procedência } \\
\hline Distrito Federal & 10 & 9 \\
\hline Outros Estados & 101 & 91 \\
\hline \multicolumn{3}{|l|}{ Prole } \\
\hline Sem filhos & 62 & 55,9 \\
\hline Com filhos & 49 & 44,1 \\
\hline \multicolumn{3}{|l|}{ Etiologia da Lesão } \\
\hline Acidentes de trânsito & 55 & 49,6 \\
\hline Acidentes por arma de fogo & 30 & 27 \\
\hline Queda de altura & 17 & 15,3 \\
\hline Queda de objeto sob o corpo & 7 & 6,3 \\
\hline Acidentes por arma branca & 2 & 1,8 \\
\hline \multicolumn{3}{|l|}{ Tipo de lesão } \\
\hline Completa & 94 & 84,7 \\
\hline Incompleta & 17 & 15,3 \\
\hline \multicolumn{3}{|l|}{ Tempo de lesão (anos) } \\
\hline Menos de 1 & 55 & 49,6 \\
\hline $1-2$ & 39 & 35,1 \\
\hline $3-4$ & 8 & 7,2 \\
\hline 5 ou mais & 9 & 8,1 \\
\hline
\end{tabular}

sentados, na amostra analisada, foram Rio Grande do Sul (22,5\%), Goiás (15,3\%), São Paulo (12,6\%) e Distrito Federal (9\%).

Com relação ao tipo de acidente, é marcante a presença da violência urbana como causa das lesões medulares traumáticas. Nos indivíduos estudados, foram observados, como principal causa de trauma, os acidentes de trânsito, que incluíram os acidentes de motocicleta (25,3\%), acidentes automobilísticos (21,6\%), atropelamentos $(1,8 \%)$ e acidentes de bicicleta $(0,9 \%)$, totalizando $49,6 \%$ da população estudada. Entre os entrevistados, $84,7 \%$ das lesões medulares foram completas, ou seja, de acordo com a classificação da American Spinal Injury Association (ASIA), 94 indivíduos tiveram secção completa da medula e foram classificados como ASIA $A$.

O WHOQOL - bref é um questionário auto-explicativo de auto-avaliação. Na amostra do presente estudo, em 96 casos o questionário foi auto-administrado (86,5\%). Outros 15 indivíduos necessitaram de alguma ajuda do entrevistador, sendo que em 9 casos $(8,1 \%)$ o entrevistado foi assistido pelo entrevistador e em 6 casos $(5,4 \%)$ o questionário foi administrado pelo entrevistador.

Os escores de avaliação dos quatro domínios e das duas questões gerais (avaliação geral) do WHOQOL-bref estão resumidos na Figura 1. Os resultados da Análise de Variância (Tabela 2) e do Teste de Turkey (Tabela 3) permitiram observar que existem diferenças estatisticamente significantes entre dois grupos. O primeiro é composto pelos domínios meio ambiente e físico, que obtiveram os piores escores de avaliação. O segundo, que obteve os escores mais elevados na avaliação, é composto pelos domínios psicológico e de relações sociais. Contudo, não existem diferenças estatisticamente significativas ao se comparar os domínios meio ambiente e físico entre si. O mesmo fato ocorre na comparação das dimensões psicológica e de relações sociais.

As Figuras 2, 3, 4 e 5 permitem observar as médias das avaliações obtidas nas 


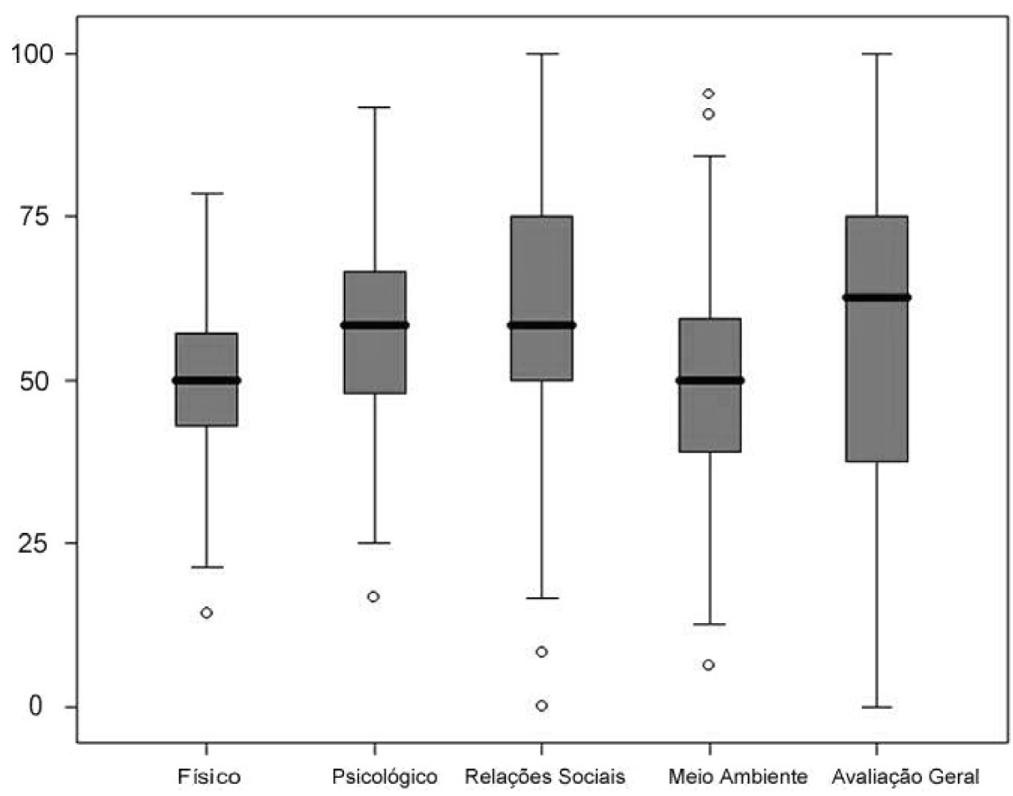

Figura 1- Escores de avaliação dos dominios que compõem o WHOQOL-bref Figure 1-Evaluation scores for the WHOQOL-bref domains

Tabela 2 - Análise de Variância entre os domínios do WHOQOL-bref Table 2 - Analysis of Variance for WHOQOL-bref domains

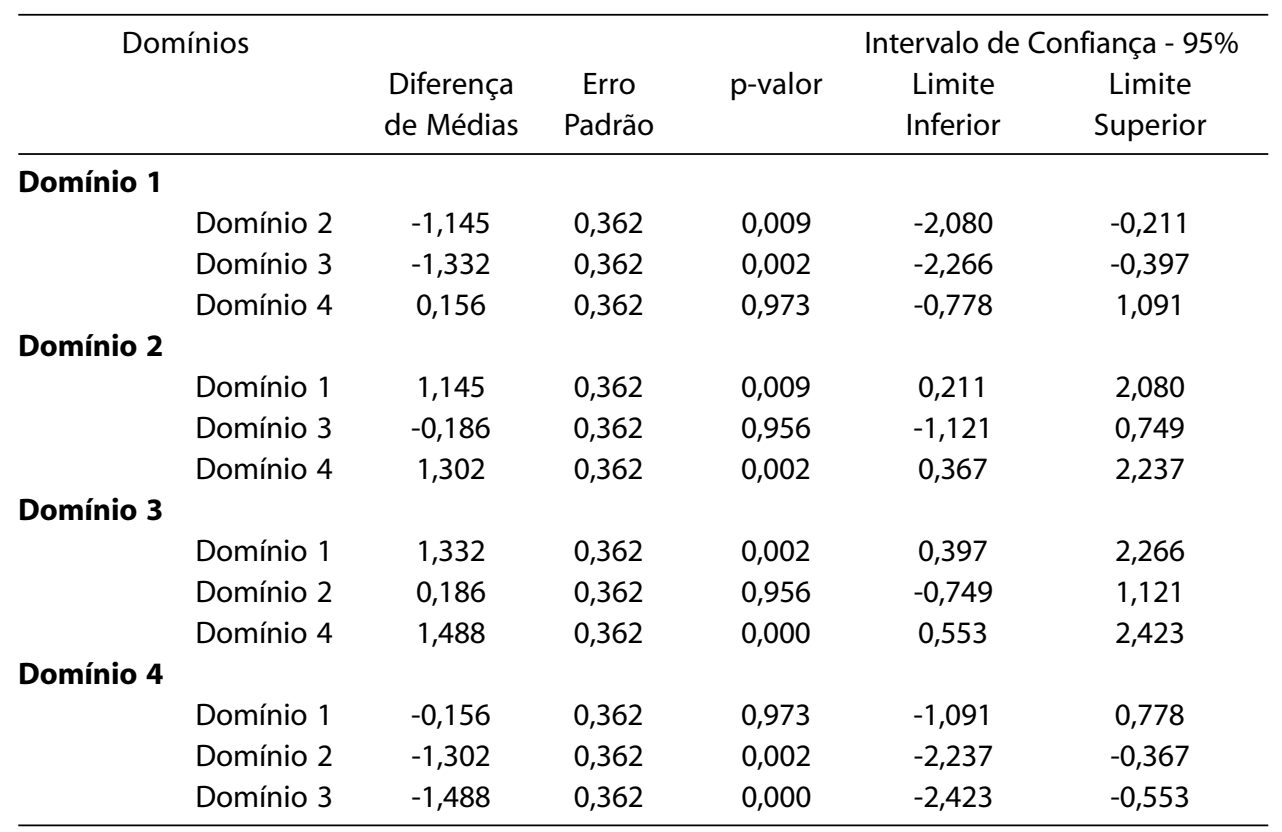

facetas que compõem os domínios do WHOQOL - bref. Esse instrumento aponta 1 como a pior resposta e 5 a melhor resposta. Para efeito de uniformização e possibilitar a comparação, as médias apresentadas nas facetas relacionadas a dor e desconforto, dependência de tratamentos ou de medicamentos e sentimentos negativos foram invertidas, conforme orientação da OMS.

No que diz respeito ao domínio físico (Figura 2), observa-se que os entrevistados 
Tabela 3 - Resultado do Teste de Turkey entre os domínios do WHOQOL-bref

Table 3 - Results of the Turkey Test for WHOQOL-bref domains

\begin{tabular}{lccc}
\hline Domínios & $\mathrm{N}$ & 1 & 2 \\
\hline Domínio 4 & 111 & 11,905 & \\
Domínio 1 & 111 & 12,062 & \\
Domínio 2 & 111 & & 13,207 \\
Domínio 3 & 111 & & 13,393 \\
Sig. & & 0,973 & 0,956 \\
\hline
\end{tabular}

relatam possuir energia para realizar as atividades do dia-a-dia e ter seu sono preservado. Contudo, estão insatisfeitos com a capacidade para o trabalho e sua mobilidade reduzida.

O domínio psicológico (Figura 3) demonstra que os entrevistados se apóiam em crenças pessoais, espiritualidade e religião, aceitam sua aparência física mantendo a auto-estima e a capacidade de pensar, aprender e concentrar-se.

Com relação ao domínio de relacionamento social (Figura 4), os indivíduos revelam receber apoio da família e dos amigos, mas apresentam problemas com a vida sexual.
Já no domínio do meio-ambiente, os problemas estão relacionados à falta de recursos financeiros, de oportunidades de recreação e lazer e de obtenção de novas informações e de habilidades, assim como dificuldades com o acesso aos meios de transporte.

\section{Discussão}

O instrumento de avaliação de qualidade de vida da OMS permitiu conhecer a percepção da qualidade de vida de pessoas com lesão medular traumática. Outra pesquisa desenvolvida na China validou esse instrumento para a pesquisa de qualidade de vida em uma população com características semelhantes às dos participantes do presente estudo em comparação com a população geral ${ }^{18}$.

Os resultados do presente estudo evidenciaram que os domínios ligados ao meio ambiente e à saúde física obtiveram os piores escores de avaliação $(49,61 \%$ e $50,26 \%$, respectivamente). Isso pode estar relacionado ao fato de a lesão medular causar alterações físicas com as quais a pessoa tem que aprender a viver. Na amostra

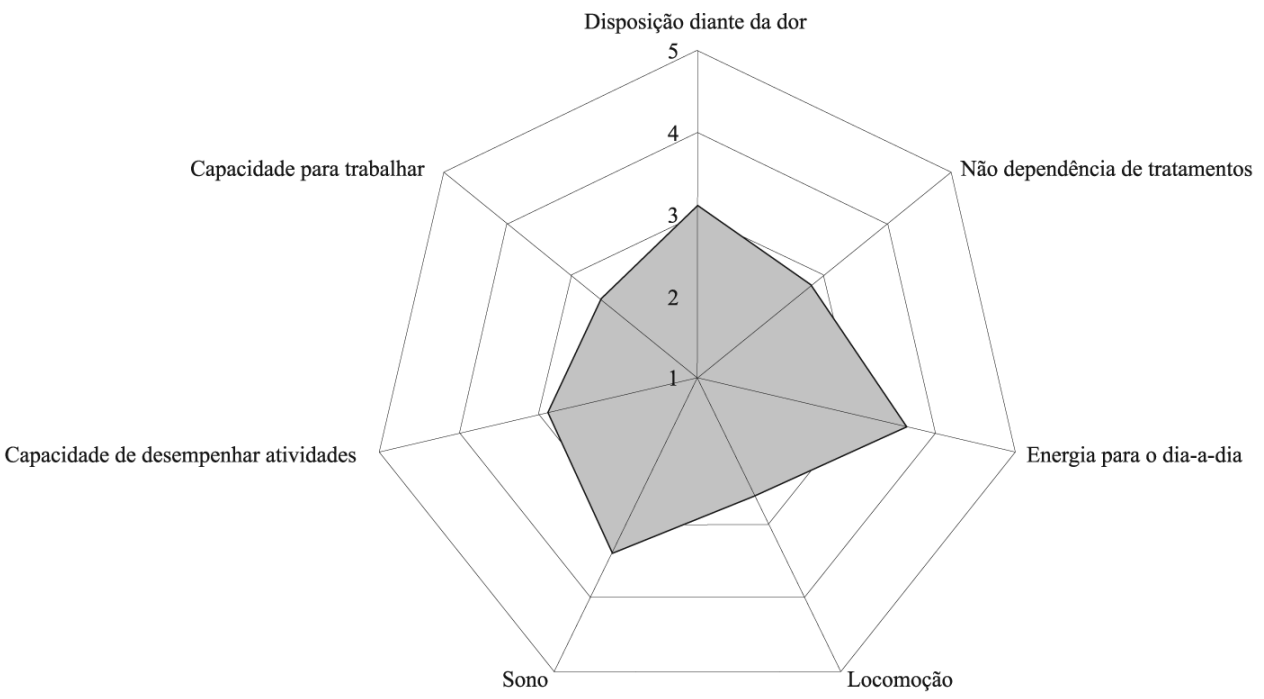

Figura 2 - Média das avaliações dos entrevistados nas facetas do domínio físico do WHOQOLbref

Figure 2 - Average of the interviewed subjects' evaluations for the facets of WHOQOL-bref physical domain 


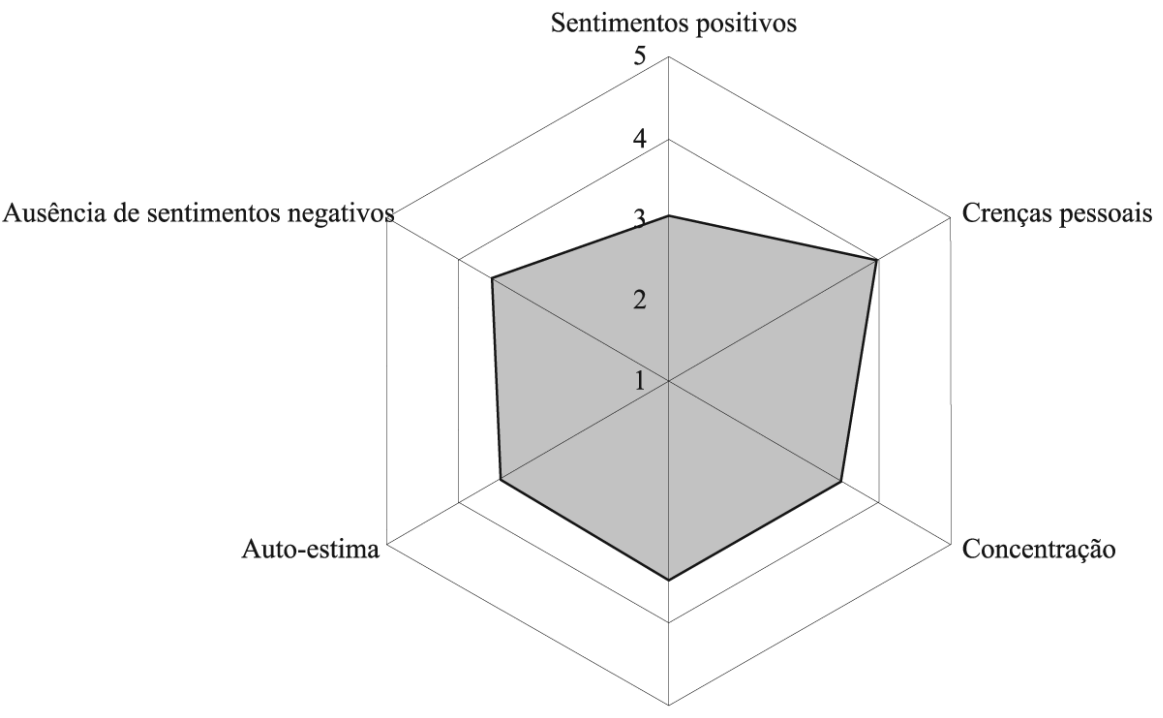

Aceitação da aparência física

Figura 3 - Média das avaliações dos entrevistados nas facetas do domínio psicológico do WHOQOL-bref

Figure 3 - Average of interviewed subjects' evaluations for the facets of WHOQOLbref psychological health domain

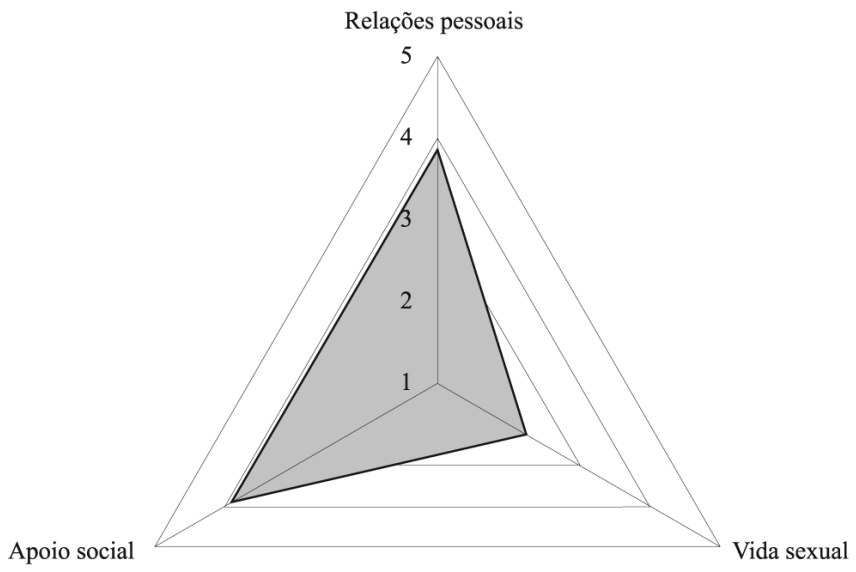

Figura 4 - Média das avaliações dos entrevistados nas facetas do domínio relações sociais do WHOQOL-bref

Figure 4 - Average of interviewed subjects' evaluations for the facets of WHOQOL-bref relationships domain

estudada, observou-se que a maioria dos entrevistados possuía pouco tempo desde o trauma e, talvez por esse motivo, ainda não lidava adequadamente com essas alterações. As mudanças físicas, assim como as barreiras sociais, podem dificultar a vida e interferir na avaliação da qualidade de vida das pessoas com lesão medular.

Uma das dificuldades encontradas na pesquisa esteve relacionada à possibilidade de se comparar os dados encontrados com resultados prévios. A literatura é muito restrita quanto a estudos que avaliam qualidade de vida em pessoas com lesão medular, especialmente utilizando a metodologia adotada pela OMS. Essa metodologia já foi empregada, no Brasil, em estudos com pessoas dependentes de 


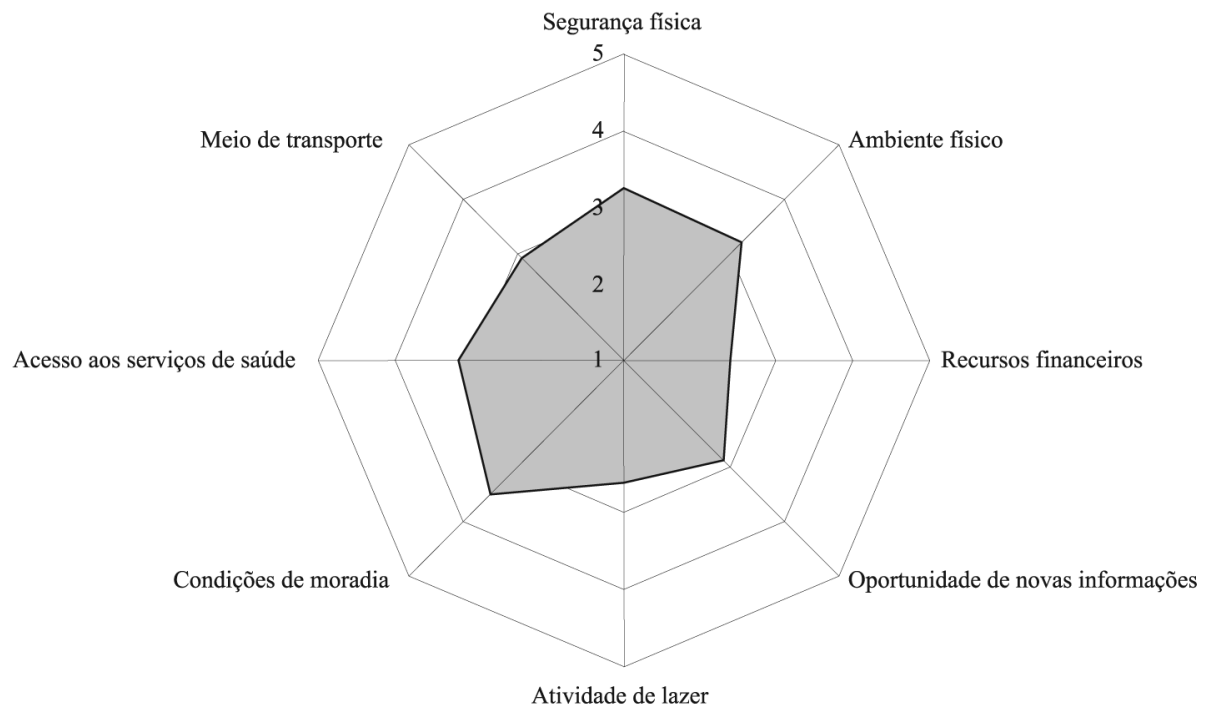

Figura 5 - Média das avaliações dos entrevistados nas facetas do domínio meio ambiente do WHOQOL-bref

Figure 5 - Average of interviewed subjects' evaluations for the facets of WHOQOL-bref environment domain

álcool ${ }^{8}$, com depressão $0^{12}$ e em idosos ${ }^{15}$.

Ao analisar estudos sobre a avaliação da qualidade de vida em indivíduos com lesão medular, mesmo com o uso de outras ferramentas de pesquisa, pode-se verificar que, tanto em estudos nacionais como em outros países, os escores de avaliação de qualidade de vida são considerados baixos ${ }^{14,18-22}$.

Em estudo desenvolvido nos Estados Unidos $^{20}$, com 215 portadores de lesão medular traumática aguda, utilizando o instrumento SF-36, os autores verificaram a correlação entre o nível de lesão medular e a avaliação dos aspectos físicos e mentais da qualidade de vida. Encontraram que houve correlação apenas entre o componente físico e o grau de incapacidade. Naquele estudo, verificou-se que, quanto maior for o comprometimento físico decorrente da lesão, menor será o escore de avaliação do domínio físico na avaliação da qualidade de vida. No presente estudo, cuja população em sua maioria tem menos de um ano de lesão, também se observou que o domínio físico foi um dos que recebeu escore mais baixo de avaliação.

A literatura aponta para um comprometimento da qualidade de vida das pes- soas com lesão da medula espinhal. No entanto, os estudos diferem em relação aos domínios mais comprometidos, de acordo com o instrumento empregado e a amostra estudada. Em algumas pesquisas, as relações sociais estão mais comprometidas $^{14,23}$. Em outros, os fatores ambientais comprometeram de forma importante a qualidade de vida das pessoas ${ }^{24}$, principalmente no que diz respeito à participação social, como é o caso do presente estudo. Nesse sentido, uma revisão na literatura sobre qualidade de vida e lesão medular, no período de 1990 e 2003, observou que a maioria dos estudos mostrava uma insatisfação com a vida após a lesão, e essa insatisfação estava relacionada principalmente às desvantagens sociais que o indivíduo passava a enfrentar ${ }^{25}$.

Já estudo realizado no Canadá, com 587 portadores de lesão medular, membros do Quebec Paraplegic Association, concluiu que idade, emprego e hospitalização são fatores que interferem na inserção social dessa população, assim como em sua qualidade de vida ${ }^{21}$.

A exemplo do que ocorreu no presente estudo, pesquisa realizada nos Estados Unidos com pessoas com lesão medular 
afro-americanas também demonstrou ser a vida sexual, o emprego e os recursos financeiros algumas das facetas mais afetadas na avaliação da qualidade de vida dos entrevistados ${ }^{26}$.

O presente estudo permitiu conhecer a avaliação da qualidade de vida de pessoas com lesão da medula espinhal. Espera-se que esse conhecimento permita uma valorização das percepções dessa população em relação às diversas áreas de sua vida e, desse modo, aspectos prioritários para o tratamento e reabilitação possam ser identificados e alcançados. Além disso, espera-se um envolvimento maior da sociedade na busca de ações que melhorem a qualidade de vida desses indivíduos.
São necessários ainda estudos mais detalhados nessa área. Poderiam ser construídos e validados instrumentos específicos de pesquisa para avaliação da qualidade de vida nessa população. Também seria importante incluir nas pesquisas indivíduos tetraplégicos e com lesões medulares não traumáticas, com o intuito de se obter uma avaliação mais global. Outra vertente a ser explorada poderiam ser pesquisas durante e após o programa de reabilitação para verificar se ocorreram mudanças na percepção da qualidade de vida. Paralelamente, é possível a utilização de metodologias qualitativas, pois qualidade de vida é um tema subjetivo e as metodologias poderiam ser complementares.

\section{Referências}

1. Lianza S, Casalis ME, Greve JMD, Eichberg R. A lesão medular. In: Lianza S (Org.). Medicina de Reabilitação. Rio de Janeiro: Guanabara Koogan; 2001. p. 299-322.

2. Doward LC, McKenna SP. Evolution of quality of life assessment. In: Rajagopalan R, Sheretz EF, Anderson RT (Ed.). Care Management of Skin Diseases: life quality and economic impact. New York: Marcel Dekker; 1997. p. 933.

3. Schipper H, Clinch J, Olweny C. Quality of life studies: definitions and conceptual issues. In: Spiker B (Edi.). Quality of Life and Pharmaeconomics in clinical trials. 2a ed. Philadelphia: Lippincott-Raven; 1996. p. 11-23.

4. The WHOQOL Group. The development of the World Health Organization quality of life assessment instrument (WHOQOL). In: Orley J, Kuyken W (Ed.). Quality of life assessment: international perspectives. Heidelberg: Springer Verlag; 1994. p. 41-60.

5. The WHOQOL Group. The World Health Organization quality of life assessment (WHOQOL): development and general psychometric properties. Soc Sci Méd 1998; 46 (12): 1569- 85.

6. Fleck M et al. Desenvolvimento da versão em português do instrumento de avaliação de qualidade de vida da Organização Mundial de Saúde (WHOQOL-100). Rev Bras Psiquiatr 1999; 21(1): 19-28.

7. Fleck M et al. Aplicação da versão em português do instrumento abreviado de avaliação de qualidade de vida "WHOQOL-bref". Rev Saúde Pública 2000; 34(2): 178-83.
8. Lima AFBS. Qualidade de vida em pacientes do sexo masculino dependentes de álcool [dissertação de mestrado]. Porto Alegre: Universidade Federal do Rio Grande do Sul; 2001.

9. Fleck M et al. Long-stay patients in a psychiatric hospital in southern Brazil. Rev Saúde Pública 2007; 41(1): 124-30.

10. Seidl EM, Zannon CM, Tróccoli BT. Pessoas vivendo com HIV/AIDS: enfren-tamento, suporte social e qualidade de vida. Psico: Reflexão e Crítica. 2005; 18(2): 188-95.

11. Castro MG et al. Qualidade de vida e gravidade da dependência de tabaco. Rev Psiq Clín 2007; 34(2): 61-7.

12. Fleck M et al. Associação entre sintomas depressivos e funcionamento social em cuidados primários de saúde. Rev Saúde Pública 2002; 36(4): 431-8.

13. Roque, VM, Forones, NM. Avaliação da qualidade de vida e toxicidades em pacientes com câncer colorretal tratados com quimioterapia adjuvante baseada em fluoropirimidinas. Arq Gastroenterol 2006; 43(2): 94-101.

14. Vall J, Braga VAB, Almeida PC. Estudo da qualidade de vida em pessoas com lesão medular traumática. Arq Neuro-Psiquiatr 2006; 64(2b): 451-5.

15. Pereira RJ, Cotta RMM, Franceschini SCC, Ribeiro RCL, Sampaio RF, Priore SE e Cecon PR. Contribuição dos domínios físico, social, psicológico e ambiental para a qualidade de vida global de idosos. Rev Psiquiatr 2006; 28(1): 27-38. 
16. Rede Sarah de Hospitais do Aparelho Locomotor. Relatório da Gestão 2001-04. Brasília: Centro Nacional de Controle de Qualidade; 2001-04.

17. Brasil, Ministério da Saúde. Conselho Nacional de Ética em Pesquisa. Diretrizes e Normas Reguladoras de Pesquisa Envolvendo Seres Humanos. Resolução 196/96. Disponível em URL: http://conselho.saude.gov.br [Acessado em julho de 2006].

18. Jang Y, Hsieh CL, Wang YH, Wu YH. A validity study of the WHOQOL-bref assessment in persons with traumatic spinal cord injury. Arch Phys Med Rehabil 2004; 85: 18905.

19. Dahlberg A, Alaranta H, Sintonen H. Health-related quality of life in persons with traumatic spinal cord lesion in Helsinki. J Rehabil Med 2005; 37: 312-6.

20. Forchheimer M, McAweeney M, Tate DG. Use of SF-36 among persons with spinal cord injury. Am J Phys Med Rehabil 2004; 83: 390-5.

21. Leduc BE, Lepage Y. Health-related quality of life after spinal cord injury. Disabil Rehabil 2002; 24(4): 196-202.
22. Unalan H. Quality of life of primary caregivers of spinal cord injury survivors living in the community: controlled study with SF-36. Spinal Cord 2001; 396: 318-22.

23. Kreuter M, Siosteen A, Erkholm B, Bystrom U, Brown DJ. Health and quality of life of persons with spinal cord lesion in Australian and Sweden. Spinal Cord 2005; 43: 123-9.

24. Whiteneck G, Meade MA, Dijkers M, Tate DG, Bushnik T, Forchheimer M. Environmental factors and their role in participation and life satisfaction after spinal cord injury. Arch Phys Med Rehabil 2004; 85: 1793-803.

25. Hammell KW. Exploring quality of life following high spinal cord injury: a review and critique. Spinal Cord 2004; 42: 491-502.

26. Krause JS, Lynne EB, Broyles J. Subjective well-being among African-American with spinal cord injury: an exploratory study between men and women. Neuro Rehabil 2004; 19: 81-9.

Recebido em: 27/02/07

Versão final reapresentada em: 22/10/07

Aprovado em: 04/12/07 